Vascular lesions of the syndrame (angiomatosis retinae and haemangioblastama of the CNS) occurred in most families and represented core features whereas phaeochromocytoma at one end of the scale, and renal lesions, pancreatic cysts and epididymal cystadenoma at the other end of the scale, were unlikely to develop in the same family and were mutually exclusive. The authors suggest that HLS is caused from different mutations within a complex genetic locus, or additional genetic lesions, which cooperate with the HLS gene on chromosome 3p. The data point to a linear sequence of features. The calculated prevalence of the disease in the district of Freiburg, Germany, with a population of $1.909 \mathrm{million}$, was 1/38951. (Neumann HPH, Wiestler OD. Clustering of features of von Hippel-Lindau syndrome: evidence for a complex genetic locus. Lancet May 4, 1991; 337:1052-54).

COMENT. These familial patterns of lesions are important in prognosis and the clinical management of HLS gene carriers. Patients with renal lesions are at a high risk for hemangioblastoma of the CNS, the principal cause of death in HLS patients. Patients presenting with renal disorders should be carefully monitored for the development of CNS lesions. Patients with pheochromocytoma are likely to develop angiomatosis retinae but are usually spared the CNS complication.

Von Hippel-Lindau disease has been associated with loss of a region of chromosome 3 that is often deleted in renal cell carcinoma. This region contains a tumor suppressor gene whose absence is associated with the development of renal cell carcinoma, hemangioblastoma and other tumors of von Hippel-Lindau disease. Deletion of the tumor suppressor gene may allow ectopic production of erythropoietin by the intracranial hemangioblastoma. Production of erythropoietin by a recurrent intracranial hemangioblastoma in a 59 year old woman was thought to explain the erythrocytosis in this patient with von Hippel-Lindau disease. (Horton JC et al. Von Hippel-Lindau disease and erythrocytosis: Radioimmunoassay of erythropoietin in cyst fluid from a brainstem hemangioblastama. Neurology May 1991; 41:753-754).

\title{
NEUROMUSCULAR DISORDERS
}

\section{MUSCLE HYPOTONIA IN LONE'S SYNDROME}

As part of a comprehensive evaluation of 23 patients with the oculocerebrorenal syndrame of Lowe at the Section on Human Biochemical Genetics, National Institutes of Health, Bethesda, MD, concentrations of muscle enzymes and carnitine metabolism were studied in addition to special reference to growth and renal function. The diagnostic findings included congenital cataracts, neurologic involvement (cognitive impairment, areflexia, and a history of infantile hypotonia), and renal tubular dysfunction in male patients with typical facies (deep set eyes and frontal bossing). Patients ranged in age from four months to 31 years. Muscle involvement was indicated by an elevation of serum 
creatine kinase concentration in conjunction with substantial concentrations of MB isoenzymes, elevated aspartate aminotransferase and lactate dehydrogenase in the setting of normal liver function. One-third of the patients had plasma free carnitine concentration below $20 \mathrm{mamol} / \mathrm{L}$, a concentration associated with depletion of carnitine in tissues. Linear growth decreased after one year of age and head circumferences, normal in the first three to four years of life, then fell to the third percentile in mid-childhood. Longevity was reduced because of renal disease and the oldest patient examined was 33 years. (Charnas LR, Gahl WA et al. Clinical and laboratory findings in the oculocerebrorenal syndrome of Lowe, with special reference to growth and renal function. N Engl J Med May 9, 1991; 324:1318-25).

COMMENT. The oculocerebrorenal syndrome of Lowe, an $\mathrm{X}$-linked recessive disorder, is a rare cause of neonatal hypotonia or the limp infant syndrome. Both muscle and central abnormalities may contribute to the hypotonia and areflexia.

\section{PREDNISONE-TREATED DUCHENNE MUSCULAR DYSTROPHY}

The immosuppressant/antiinflammatory effects of prednisone were studied in 33 patients with Duchenne muscular dystrophy (ages 5 to 15 years) at the conclusion of a six month treatment trial at the Department of Neurology, Division of Neuromuscular Disease, Ohio State University College of Medicine, Columbus, OH. Immunohistochemical analyses were carried out on muscle biopsies: 12 from the placebo group, 9 from the low-dose prednisone group $(0.75 \mathrm{mg} / \mathrm{kg} / \mathrm{d})$, and 12 from the high-dose group $(1.5 \mathrm{mg} / \mathrm{kg} / \mathrm{d})$. The number of $\mathrm{T}$ cells and the number of muscle fibers focally invaded by $\mathrm{T}$ cells were significantly decreased in the prednisone treated groups compared with controls. Prednisone may improve strength in Duchenne muscular dystrophy through primarily immunologic mechanisms involving $\mathrm{T}$ lymphocytes. (Kissel JT et all. Mononuclear cell analysis of muscle biopsies in prednisone-treated and untreated Duchenne muscular dystrophy. Neurolo-

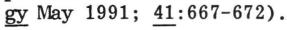

COMMENT. The possibility that clinical improvement was related to prednisone-induced alterations in skeletal muscle dystrophin was disproved by a further study from Ohio State University (Burrow $\mathrm{KL}$ et al. Dystrophin expression and somatic reversion in prednisone-treated and untreated Duchenne dystrophy. Neurology May $1991 ; 41: 661-666)$. Dystrophin content was analyzed at the conclusion of a six month trial of prednisone, using Western blots and antibody staining of tissue sections. There was no significant differences in dystrophin content between treatment and placebo groups.

\section{INFECTIOUS DISEASES}

\section{EPIDEMIOLOGY OF ENCEPHALITIS}

The incidence and epidemiology of encephalitis in children have been analyzed in a 20 year survey at the Department of Virology and the 